\title{
Common misconceptions about data analysis and statistics
}

\author{
Harvey J. Motulsky
}

Received: 8 August 2014 / Accepted: 14 August 2014 / Published online: 12 September 2014

(C) The Author(s) 2014. This article is published with open access at Springerlink.com

\begin{abstract}
Ideally, any experienced investigator with the right tools should be able to reproduce a finding published in a peerreviewed biomedical science journal. In fact, the reproducibility of a large percentage of published findings has been questioned. Undoubtedly, there are many reasons for this, but one reason maybe that investigators fool themselves due to a poor understanding of statistical concepts. In particular, investigators often make these mistakes: 1. P-Hacking. This is when you reanalyze a data set in many different ways, or perhaps reanalyze with additional replicates, until you get the result you want. 2. Overemphasis on $P$ values rather than on the actual size of the observed effect. 3. Overuse of statistical hypothesis testing, and being seduced by the word "significant". 4. Overreliance on standard errors, which are often misunderstood.
\end{abstract}

\section{Introduction}

Ideally, any experienced investigator with the right tools should be able to reproduce a finding published in a peerreviewed biomedical science journal. In fact, the reproducibility of a large percentage of published findings has been questioned. Investigators at Bayer Healthcare were reportedly

This commentary evolved from multiple conversations between the author and editors of several pharmacology journals. This work represents solely the opinions of the author. Publication of this article does not represent an endorsement of GraphPad Software. This article is being simultaneously published in Br J Pharmacol, J Pharmacol Exp Ther, Naunyn-

Schmiedeberg's Arch Pharmacol, and Pharmacol Res Perspect in a collaborative effort to help investigators and readers to appropriately use and interpret statistical analyses in pharmacological research studies. This work is licensed under a Creative Commons Attribution-NoDerivs 4.0 International (CC BY-ND 4.0) license (https://creativecommons.org/ licenses/by-nd/4.0/legalcode). doi: 10.1007/s00210-014-1037-6.

H. J. Motulsky $(\bowtie)$

GraphPad Software Inc., La Jolla, California, USA

e-mail: hmotulsky@graphpad.com able to reproduce only 20-25\% of 67 preclinical studies (Prinz et al. 2011), and investigators at Amgen were able to reproduce only 6 of 53 studies in basic cancer biology despite often cooperating with the original investigators (Begley and Ellis 2012). This problem has been featured in a cover story in The Economist (Anonymous 2013) and has attracted the attention of the NIH leaders (Collins and Tabak 2014).

Why can so few findings be reproduced? Undoubtedly, there are many reasons. But in many cases, I suspect that investigators fooled themselves due to a poor understanding of statistical concepts (see Marino 2014, for a good review of this topic). Here, I identify five common misconceptions about statistics and data analysis, and explain how to avoid them. My recommendations are written for pharmacologists and other biologists publishing experimental research using commonly used statistical methods. They would need to be expanded for analyses of clinical or observational studies and for Bayesian analyses. This editorial is about analyzing and displaying data, so it does not address issues of experimental design.

My experience comes from basic pharmacology research conducted decades ago, followed by 25 years of answering email questions from scientists needing help analyzing data with GraphPad Prism, ${ }^{1}$ and authoring three editions of the text Intuitive Biostatistics (Motulsky 2014a).

\section{Misconception 1: P-Hacking is $\mathrm{OK}$}

Statistical results can only be interpreted at face value when every choice in data analysis was performed exactly as planned and documented as part of the experimental design. From my conversations with scientists, it seems that this rule is commonly broken in reports of basic research. Instead, analyses are often done as shown in Fig. 1. Collect and analyze

\footnotetext{
${ }^{1}$ http://www.graphpad.com/prism
} 


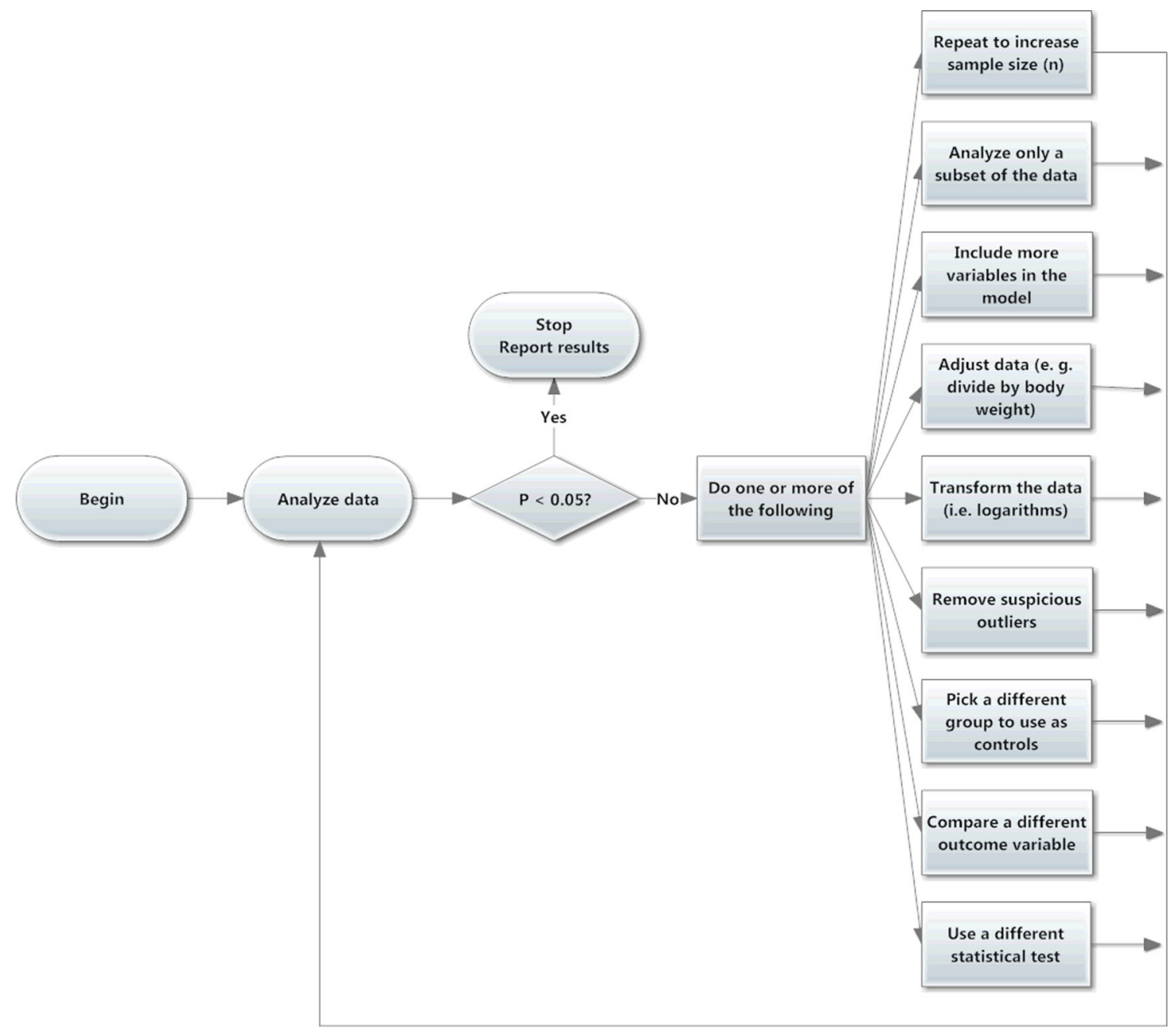

Fig. 1 The many forms of P-hacking. When you P-hack, the results cannot be interpreted at face value. Not shown in the figure is that after trying various forms of P-hacking without getting a small $P$ values, you will eventually give up when you run out of time, funds, or curiosity

some data. If the results are not statistically significant but show a difference or trend in the direction you expected, collect some more data and reanalyze. Or try a different way to analyze the data: remove a few outliers; transform to logarithms; try a nonparametric test; redefine the outcome by normalizing (say, dividing by each animal's weight); use a method to compare one variable while adjusting for differences in another; the list of possibilities is endless. Keep trying until you obtain a statistically significant result or until you run out of money, time, or curiosity.

The results from data collected this way cannot be interpreted at face value. Even if there really is no difference (or no effect), the chance of finding a "statistically significant" result exceeds $5 \%$. The problem is that you introduce bias when you choose to collect more data (or analyze the data differently) only when the $P$ value is greater than 0.05 . If the $P$ value was less than 0.05 in the first analyses, it might be larger than 0.05 after collecting more data or using an alternative analysis. But you would never see this if you only collected more data or tried different data analysis strategies when the first $P$ value was greater than 0.05 .
Exploring your data can be a very useful way to generate hypotheses and make preliminary conclusions. But all such analyses need to be clearly labeled, and then retested with new data.

There are three related terms that describe this problem:

- Ad hoc sample size selection. This is when you did not choose a sample size in advance, but just kept going until you liked the results. Figure 2 demonstrates the problem with ad hoc sample size determination. Distinguish unplanned ad hoc sample size decisions from planned "adaptive" sample size methods that make you "pay" for the increased versatility in sample size collection by requiring a stronger effect to reach "significance" (Kairalla et al. 2012; FDA 2010).

- Hypothesizing after the result is known (HARKing; Kerr 1998). This is when you analyze the data in many different ways (say different subgroups), discover an intriguing relationship, and then publish the data so it appears that the hypothesis was stated before the data were collected (Fig. 3). This is a form of multiple comparisons (Berry 2007). Kriegeskorte et al. (2009) call this double dipping, 


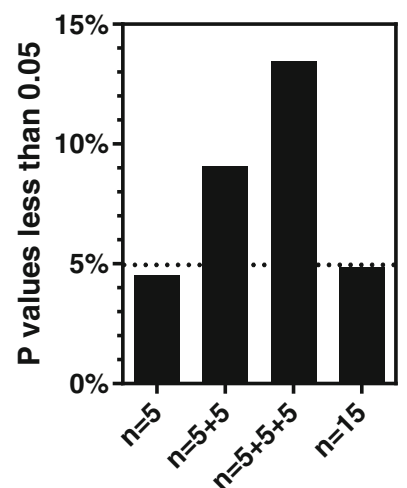

Fig. 2 The problem of ad hoc sample size selection. I simulated 10,000 experiments sampling data from a Gaussian distribution with means of 5.0 and standard deviations of 1.0, and comparing two samples with $n=5$ each using an unpaired $t$ test. The first column shows the percentage of those experiments with a $P$ value less than 0.05 . Since both populations have the same mean, the null hypothesis is true and so (as expected) about $5.0 \%$ of the simulations have $P$ values less than 0.05 . For the experiments where the $P$ value was higher than 0.05 , I added five more values to each group. The middle column (" $n=5+5$ ") shows the fraction of $P$ values where the $P$ value was less than 0.05 either in the first analysis with $n=5$ or after increasing the sample size to 10 . For the third column, I added yet another 5 values to each group if the $P$ value was greater than 0.05 for both of the first two analyses. Now $13 \%$ of the experiments (not $5 \%$ ) have reached a $P$ value less than 0.05 . For the fourth column, I looked at all 10,000 of the simulated experiments with $n=15$. As expected, very close to $5 \%$ of those experiments had $P$ values less than 0.05 . The higher fraction of "significant" findings in the $n=$ $5+5$ and $n=5+5+5$ is due to the fact that I increased sample size only when the $P$ value was high with smaller sample sizes. In many cases, when the $P$ value was less than 0.05 with $n=5$, the $P$ value would have been higher than 0.05 with $n=10$ or 15 , but an experimenter seeing the small $P$ value with the small sample size would not have increased sample size

as you are using the same data both to generate a hypothesis and to test it.

- $P$-hacking. This is a general term that encompasses dynamic sample size collection, HARKing, and more. It was coined by Simmons et al. (2011) who also use the phrase, "too many investigator degrees of freedom." P-hacking is especially misleading when it involves changing the actual values analyzed. Examples include ad hoc sample size selection (see above), switching to an alternate control group (if you do not like the first results and your experiment involved two or more control groups), trying various combinations of independent variables to include in a multiple regression (whether the selection is manual or automatic), and analyzing various subgroups of the data. Reanalyzing a single data set in various ways is also Phacking but will not usually mislead you quite as much.

My suggestion for authors:

- For each figure or table, clearly state whether or not the sample size was chosen in advance, and whether every step used to process and analyze the data was planned as part of the experimental protocol.

- If you use any form of P-hacking, label the conclusions as "preliminary."
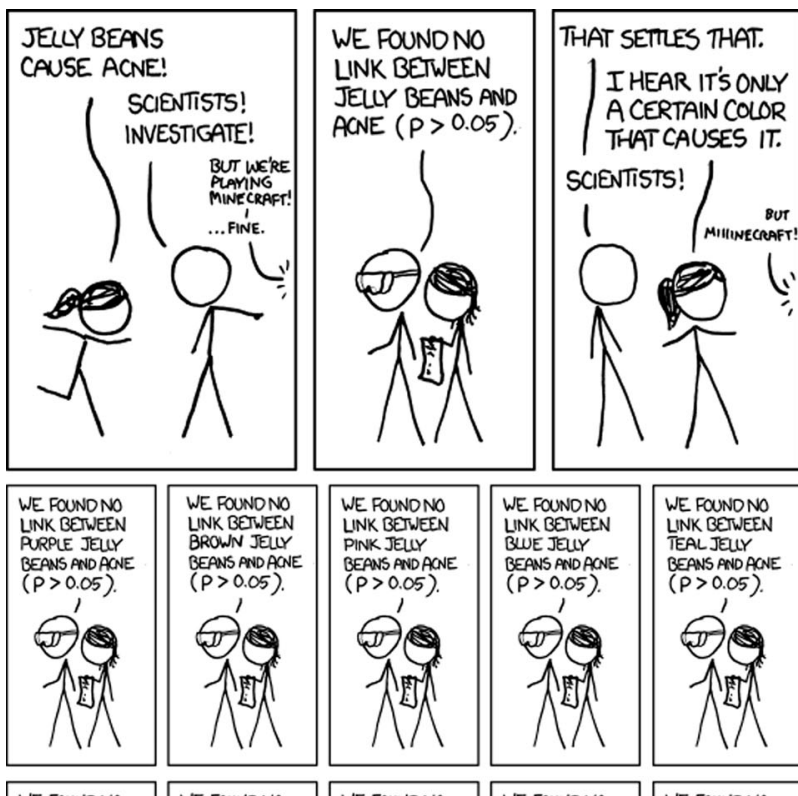

WE FOUNDNO

LINK BETWEEN

SEANS AND ACN

$P>0.05$ )

WE FOUNDNO

SALMON JELY

BEANS PND AONE

$(P>0.05)$.

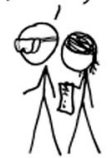

WE FOUNONO
UNK BETWEEN

RED JEUY

BEANS AND AONE

$(p>0.05)$.

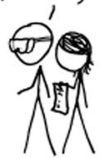

WE FOUNONO

LINK BETWEEN

BEPNS AND ACNE

$(P>0.05)$.
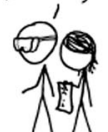

N

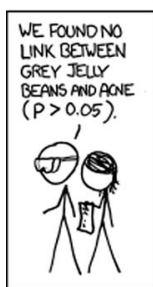

WE FOUNONO

LINK BETWEEN

TAN JELYY
BEANS AND AOIE

BEANS AND AONE
$(P>0.05)$.

\section{WE FOUNONO}

LINK BETEEN

CYAN JELUY

BEANS AND AONE
$(P>0.05)$.
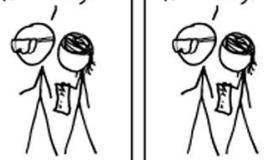

WE FOUNONN
LINK BETWEE
MAGENTA JEUY

MAGENTA JEUYY

BEANS AND PANE
$(P>0.05)$

$(p>0.05)$.

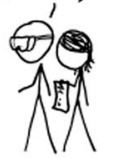

TEAL JeUIY

BEANS PND AONE

$(p>0.05)$.



WE FOUNOND

LINK BETEEE

BEIGE JELUY

BEENS AND AONE
$(p>0.05)$.

$P>0.05$ )

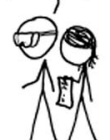

WE FOUNO NO

UINK BETWEEN

ULAC JELUY

BEANS AND AO
$(P>0.05)$.

WE FOUNONO

LINK BETWEEN

BLACK JELYY

BEANS AND AONE
$(P>0.05)$.

WE FOUNO A

GREEN JELYY

BEANS PND FANE

WHOA!

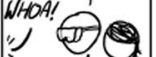

Non

影

WE FOUNONO

LINK BETWEEN

PEACH JELUY
BEANS AND PNE

BEANS AND AONE
$(P>0.05)$

> 0.05

WE FOUNDNO

LINK BETWEEN
YELOW JELYY

BEANS PND AON

$(p>0.05)$.
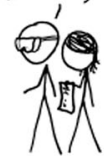

WE FOUNONO

LINK BETWEEN

MAUVE JELYY
BEANS PNO OOW

BEANS NND AON
$(p>0.05)$
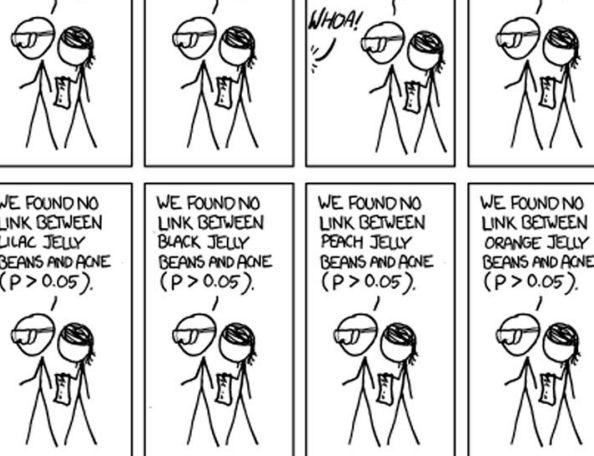

WE FOUNONO

LINK BETWEEN

ORANGE JELY

BEANS AND AON
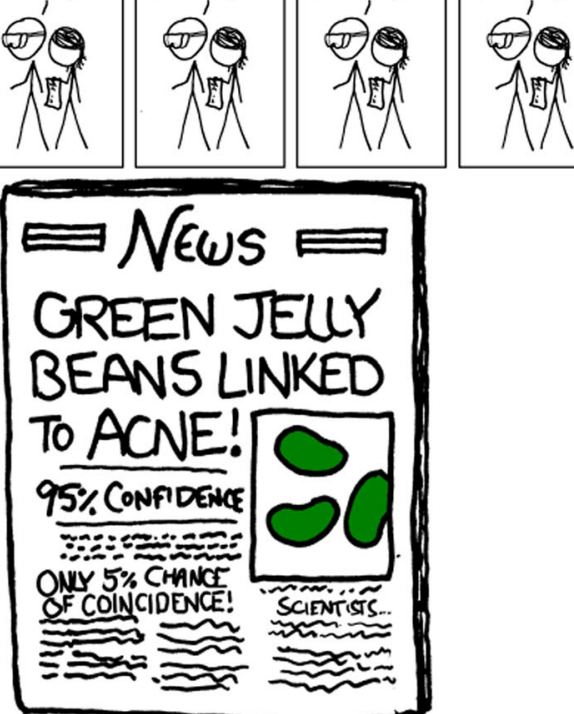

Fig. 3 The problem of hypothesizing after the results are known (HARKing) from http://xkcd.com/882/ 
Misconception 2: $P$ values convey information about effect size

To compute a $P$ value, you first must clearly define a null hypothesis, usually that two means (or proportions or $\mathrm{EC}_{50}$ 's...) are identical. Given some assumptions, the $P$ values are the probability of seeing an effect as large as or larger than you observed in the current experiment if in fact the null hypothesis was true. But note that the $P$ value gives you no information about how large the difference (or effect) is. Figure 4 demonstrates this point by plotting the $P$ values that result from comparing two samples in experiments with different sample sizes. Even though the means and standard deviations are identical for each simulated experiment, the $P$ values are far from identical. With $n=3$ in each group, the $P$ value is 0.65 . When $n=300$ in each group, the $P$ value is 0.000001 .

The dependence of $P$ values on sample size can lead to two problems.

\section{A large $P$ value is not proof of no (or little) effect}

The top two rows of Table 1 presents the results of two simulated experiments. The two $P$ values are both about 0.6 , but the two experiments lead to very different conclusions.

In experiment $\mathrm{A}$ (from Table 1), the difference between means in the experimental sample is 10 , so the difference equals $1 \%$ of the mean of treatment 1 . Assuming random sampling from Gaussian populations, the $95 \%$ confidence interval for the difference between the two population means ranges from -30 to 50 . In other words, the data are consistent (with $95 \%$ confidence) with a decrease of $3 \%$, an increase of $5 \%$, or anything in between. The interpretation depends on the scientific context and the goals of the experiment, but in most contexts, these results can be

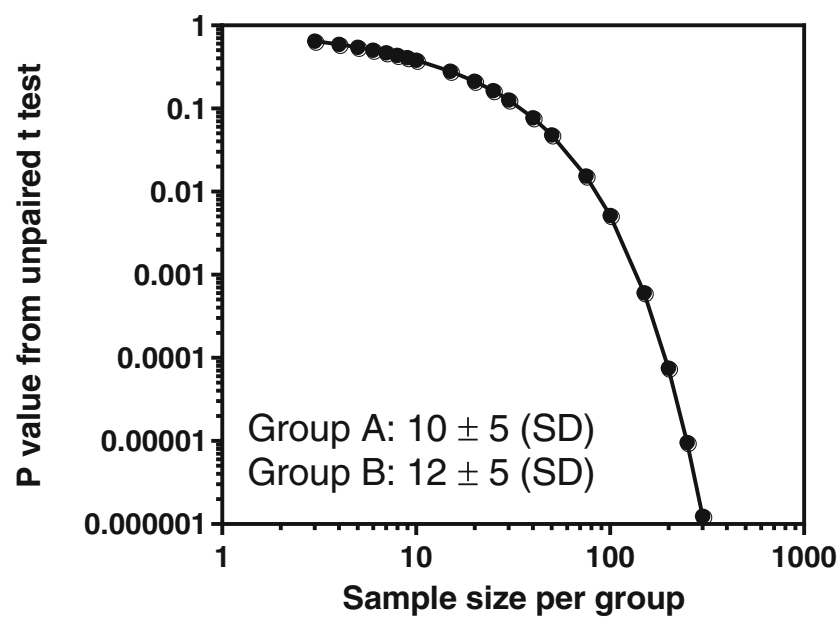

Fig. $4 P$ values depend upon sample size. This graph shows $P$ values computed by unpaired $t$ tests comparing two sets of data. The means of the two samples are 10 and 12. The SD of each sample is 5.0. I computed a $t$ test using various sample sizes plotted on the $x$-axis. You can see that the $P$ value depends on sample size. Note that both axes use a logarithmic scale summarized simply: The data are consistent with a tiny decrease, no change, or a tiny increase. These are solid negative data.

Experiment B is very different. The difference between means is larger, and the confidence interval is much wider (because the sample size is so small). Assuming random sampling from Gaussian populations, the data are consistent (with $95 \%$ confidence) with anything between a decrease of $18 \%$ and an increase of $28 \%$. The data are consistent with a large decrease, a small decrease, no difference, a small increase, or a large increase. These data lead to no useful conclusion at all! An experiment like this should not be published.

\section{A small $P$ value is not proof of a large effect}

The bottom two rows of Table 1 presents the results of two simulated experiments where both $P$ values are 0.001 , but again two experiments lead to very different conclusions.

In experiment $\mathrm{C}$ (from Table 1), the difference between means in the experimental sample is only 2 (so the difference equals $2 \%$ of the mean of treatment 1 ). Assuming random sampling from Gaussian populations, the $95 \%$ confidence interval for the difference between the two population means ranges from 0.8 to 3.2. In other words, the data are consistent (with $95 \%$ confidence) with anything between an increase of $0.8 \%$ and an increase of $3.2 \%$. How to interpret that depends on the scientific context and the goals of the experiment, but in most contexts, this can be summarized simply: The data clearly demonstrate an increase, but that increase is tiny.

Experiment D is very different. The difference between means is 35 (so $35 \%$ of the control mean), and the confidence interval extends from an increase of $23.7 \%$ to an increase of $46.3 \%$. The data clearly demonstrate that there is an increase that is (with $95 \%$ confidence) substantial.

My suggestions for authors:

- Always show and emphasize the effect size (as difference, percent difference, ratio, or correlation coefficient) along with its confidence interval.

- Consider omitting reporting of $P$ values.

\section{Misconception 3: statistical hypothesis testing and reports of "statistical significance" are necessary in experimental research}

Statistical hypothesis testing is a way to make a crisp decision from one analysis. If the $P$ value is less than a preset value (usually 0.05 ), the result is deemed "statistically significant" and you make one decision. Otherwise, the result is deemed "not statistically significant" and you make the other decision. This is helpful in quality control and some clinical studies. It also is useful when you rigorously compare the fits of two scientifically 
Table 1 Identical $P$ values with very different interpretations

\begin{tabular}{lccccc}
\hline & Treatment 1 (mean $\pm \mathrm{SD}, n)$ & $\begin{array}{l}\text { Treatment 2 } \\
(\operatorname{mean} \pm \mathrm{SD}, n)\end{array}$ & Difference between means & $\begin{array}{c}P \text { value } \\
\text { 95 \% CI of the difference } \\
\text { between means }\end{array}$ \\
\hline Experiment A & $1,000 \pm 100, n=50$ & $990.0 \pm 100, n=50$ & 10 & 0.6 & -30 to 50 \\
Experiment B & $1,000 \pm 100, n=3$ & $950.0 \pm 100, n=3$ & 50 & 0.6 & -177 to 277 \\
Experiment C & $100 \pm 5.0, n=135$ & $102 \pm 5.0, n=135$ & 2 & 0.001 & 0.8 to 3.2 \\
Experiment D & $100 \pm 5.0, n=3$ & $135 \pm 5.0, n=3$ & 35 & 0.001 & 24 to 46 \\
\hline
\end{tabular}

Experiments A and B have identical $P$ values, but the scientific conclusion is very different. The interpretation depends upon the scientific context, but in most fields experiment A would be solid negative data proving that there either is no effect or that the effect is tiny. In contrast, experiment B has such a wide confidence interval as to be consistent with nearly any hypothesis. Those data simply do not help answer your scientific question

Similarly, experiments $\mathrm{C}$ and $\mathrm{D}$ have identical $P$ values, but should be interpreted differently. In most experimental contexts, experiment $\mathrm{C}$ demonstrates convincingly that while the difference is not zero, it is quite small. Experiment $\mathrm{D}$ provides convincing evidence that the effect is large

sensible models to your data, and choose one to guide your interpretation of the data and to plan future experiments.

Here are five reasons to avoid use of statistical hypothesis testing in experimental research:

- The need to make a crisp decision based on one analysis is rare in basic research. A decision about whether or not to place an asterisk on a figure does not count! If you are not planning to make a crisp decision, the whole idea of statistical hypothesis testing is not helpful.

- Statistical hypothesis testing "does not tell us what we want to know, and we so much want to know what we want to know that, out of desperation, we nevertheless believe that it does!" (Cohen 1994). Statistical hypothesis testing has even been called a cult (Ziliak and McCloskey 2008). The question we want to answer is: Given these data, how likely is the null hypothesis? The question that a $P$ values answers is: Assuming the null hypothesis is true, how unlikely are these data? These two questions are distinct, and so have distinct answers.

- Scientists who intend to use statistical hypothesis testing often end up not using it. If the $P$ value is just a bit larger than 0.05 , scientists often avoid the strict use of hypothesis testing and instead apply the "time-honored tactic of circumlocution to disguise the nonsignificant result as something more interesting" (Hankins 2013). They do this by using terms such as "almost significant," "bordered on being statistically significant," "a statistical trend toward significance," or "approaching significance." Hankins lists 468 such phrases he found in published papers!

- The $5 \%$ significance threshold is often misunderstood. If you use a $P$ value to make a decision, of course it is possible that you will make the wrong decision. In some cases, the $P$ value will be tiny just by chance, even though the null hypothesis of no difference is actually true. In these cases, a conclusion that a finding is statistically significant is a false positive and you will have made what is called a type I error. ${ }^{2}$ Many scientists

\footnotetext{
${ }^{2}$ In contrast, a type II, or false negative, error is when there really is a difference but the result in your experiment is not statistically significant.
}

mistakenly believe that the chance of making a false positive conclusion is $5 \%$. In fact, in many situations, the chance of making a type I false positive conclusion is much higher than $5 \%$ (Colquhoun 2014). For example, in a situation where you expect the null hypothesis to be true $90 \%$ of the time (say you are screening lightly prescreened compounds, so expect $10 \%$ to work), you have chosen a sample size large enough to ensure $80 \%$ power, and you use the traditional $5 \%$ significance level, the false discovery rate is not $5 \%$ but rather is $36 \%$ (the calculations are shown in Table 2). If you only look at experiments where the $P$ value is just a tiny bit less than

Table 2 The false discovery rate when $P<0.05$

\begin{tabular}{llll}
\hline & $P<0.05$ & $P>0.05$ & Total \\
\hline Really is an effect & 80 & 20 & 100 \\
No effect (null hypothesis true) & 45 & 855 & 900 \\
Total & 125 & 875 & 1,000 \\
\hline
\end{tabular}

This table tabulates the theoretical results of 1,000 experiments where the prior probability that the null hypothesis is false is $10 \%$, the sample size is large enough so that the power is $80 \%$, and the significance level is the traditional $5 \%$. In 100 of the experiments $(10 \%)$, there really is an effect (the null hypothesis is false), and you will obtain a "statistically significant" result $(P<0.05)$ in 80 of these (because the power is $80 \%)$. In 900 experiments, the null hypothesis is true but you will obtain a statistically significant result in 45 of them (because the significance threshold is 5 and $5 \%$ of 900 is 45 ). In total, you will obtain $80+45=125$ statistically significant results, but $45 / 125=36 \%$ of these will be false positive. The proportion of conclusions of "statistical significance" that are false discoveries or false positives depends on the context of the experiment, as expressed by the prior probability (here $10 \%$ ).

If you do obtain a small $P$ value and reject the null hypothesis, you will conclude that the values in the two groups were sampled from different distributions. As noted above, there may be a high chance that you made a false positive conclusion due to random sampling. But even if the conclusion is "true" from a statistical point of view and not a false positive due to random sampling, the effect may have occurred for a reason different than the one you hypothesized. When thinking about why an effect occurred, ignore the statistical calculations, and instead think about blinding, randomization, positive controls, negative controls, calibration, biases, and other aspects of experimental design. 
0.050 , the probability of a false positive rises to $79 \%$ (Motulsky 2014b). Ioannidis (2005) used calculations like these (and other considerations) to argue that most published research findings are probably false.

- The word "significant" is often misunderstood. The problem is that "significant" has two distinct meanings in science (Motulsky 2014c). One meaning is that a $P$ value is less than a preset threshold (usually 0.05). The other meaning of "significant" is that an effect is large enough to have a substantial physiological or clinical impact. These two meanings are completely different, but are often confused.

My suggestions for authors:

- Only report statistical hypothesis testing (and place significance asterisks on figures) when you will make a decision based on that one analysis.

- Never use the word "significant" in a scientific paper. If you use statistical hypothesis testing to make a decision, state the $P$ value, your preset $P$ value threshold, and your decision. When discussing the possible physiological or clinical impacts of a finding, use other words.

\section{Misconception 4: the standard error of the mean quantifies variability}

Pharmacology journals are full of graphs and tables showing the mean and the standard error of the mean (SEM).

A quick review. The standard deviation (SD) quantifies variation among a set of values, but the SEM does not. The SEM is computed by dividing the SD by the square root of sample size. With large samples, the SEM will be tiny even if there is a lot of variability.

One problem with plotting or displaying the mean \pm SEM is that some people viewing the graph or table may mistakenly think that the error bars show the variability of the data. A second problem with reporting means with SEM is that the range mean \pm SEM cannot be rigorously interpreted. The SEM gives information about how precisely you have determined the population mean. So the range mean \pm SEM is a confidence interval, but the confidence level depends on sample size. With large samples, that range is a $68 \% \mathrm{CI}$ of the mean. When $n=3$, that range is only a $58 \% \mathrm{CI}^{3}$

My suggestions for authors:

- If you want to display the variability among the values, show raw data (which is not done often enough in my opinion). If

\footnotetext{
${ }^{3}$ Computed using this Excel formula: $=(1-$ T.DIST.2T $(1.0,2))$. The first argument (1.0) is the number of SEMs (in each direction) included in the confidence interval, and the second argument (2) is the number of degrees of freedom, which equals n-1.
}

showing the raw data would make the graph hard to read, show instead a box-whisker plot, a frequency distribution, or the mean and SD.

- If you want readers to see how precisely you have determined the mean, report a confidence interval $(95 \%$ confidence intervals are standard). Figure 5 shows a dataset plotted using all of these methods.

- When reporting results from regression, show the $95 \%$ confidence interval of each parameter rather than standard errors.

\section{Misconception 5: you do not need to report the details}

The methods section of every paper should report the methods with enough detail that someone else could reproduce your work. This applies to statistical methods just as it does to experimental methods.

My suggestions for authors:

- When reporting a sample size, explain exactly what you counted. Did you count replicates in one experiment

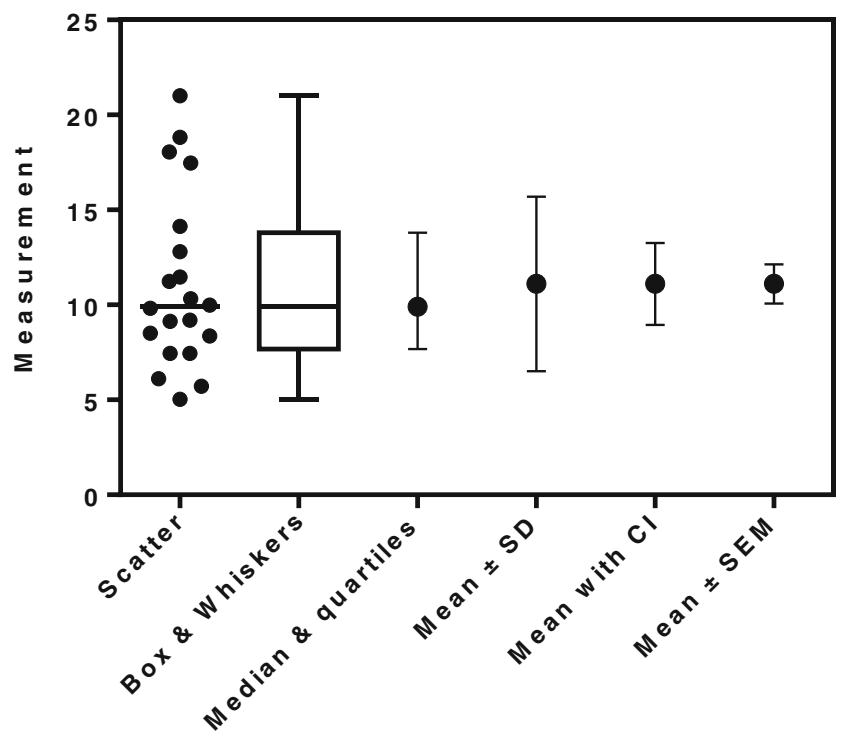

Fig. 5 Standard error bars do not show variability and do a poor job of showing precision. The figure plots one data set six ways. The leftmost lane shows a scatter plot of every value, so is the most informative. The next lane shows a box-and-whisker plots showing the range of the data, the quartiles, and the median (whiskers can be plotted in various ways, and do not always show the range). The third lane plots the median and quartiles. This shows less detail, but still demonstrates that the distribution is a bit asymmetrical. The fourth lane plots mean with error bars showing plus or minus one standard deviation. Note that these error bars are, by definition, symmetrical so give you no hint about the asymmetry of the data. The next two lanes are different than the others as they do not show scatter. Instead, they show how precisely we know the population mean, accounting for scatter and sample size. The fifth lane shows the mean with error bars showing the $95 \%$ confidence interval of the mean. The sixth (rightmost) lane plots the mean plus or minus one standard error of the mean, which does not show variation and does a poor job of showing precision 
(technical replicates), repeat experiments, the number of studies pooled in a meta-analysis, or something else?

- If you eliminated any outliers, state how many outliers you eliminated, the rule used to identify them, and a statement whether this rule was chosen before collecting data.

- If you normalized data, explain exactly how you defined 100 and $0 \%$.

- When possible, report the $P$ value up to at least a few digits of precision, rather than just stating whether the $P$ value is less than or greater than an arbitrary threshold. For each $P$ value, state the null hypothesis it tests if there is any possible ambiguity.

- When reporting a $P$ value that compares two groups, state whether the $P$ value is one- or two-tailed. If you report a one-tailed $P$ value, state that you recorded a prediction for the direction of the effect (for example increase or decrease) before you collected any data and what this prediction was. If you did not record such a prediction, report a two-tailed $P$ value.

- Explain the details of the statistical methods you used. For example, if you fit a curve using nonlinear regression, explain precisely which model you fit to the data and whether (and how) data were weighted. Also state the full version number and platform of the software you use.

- Consider posting files containing both the raw data and the analyses so other investigators can see the details.

\section{Summary}

The physicist E. Rutherford supposedly said, "If your experiment needs statistics, you ought to have done a better experiment ${ }^{4}$." There is a lot of truth to that statement when you are working in a field with a very high signal-to-noise ratio. In these fields, statistical analysis may not be necessary. But if you work in a field with a lower signal-to-noise ratio, or are trying to compare the fits of alternative models that do not differ all that much, you need statistical analyses to properly quantify your confidence in your conclusions.

I suspect that one of the reasons that the results reported in many papers cannot be reproduced is that statistical analyses are often done as a quick afterthought, with the goal to sprinkle asterisks on figures and the word "significant" on conclusions. The suggestions I propose in this commentary

\footnotetext{
$\overline{4}$ The quotation is widely attributed to this famous physicist, but I cannot find an actual citation.
}

can all be summarized simply: If you are going to analyze your data using statistical methods, then plan the methods carefully, do the analyses seriously, and report the data, methods, and results completely.

Open Access This article is distributed under the terms of the Creative Commons Attribution NoDerivatives 4.0 International License which permits re-use and distribution of the original version in any medium provided that inter alia the original author(s) and the source are credited. The license does not allow the distribution of modified versions of the article.

\section{References}

Anonymous (2013). Trouble at the lab. The Economist 409:23-27, 2013 Begley CG, Ellis LM (2012) Raise standards for preclinical cancer research. Nature 483:533

Berry DA (2007) The difficult and ubiquitous problems of multiplicities. Pharm Stat 6:155-160

Cohen J (1994) The earth is round $(p<0.05)$. Am Psychol 49:997-1003

Collins FS, Tabak LA (2014) Policy: NIH plans to enhance reproducibility. Nature 505:612-613

Colquhoun D (2014). An investigation of the false discovery rate and the misinterpretation of P values. http://arxiv.org/abs/1407.5296

FDA (2010) Guidance for Industry: Adaptive Design Clinical Trials for Drugs and Biologics. Accessed July 29, 2014 at http://www.fda.gov/ downloads/Drugs/GuidanceComplianceRegulatoryInformation/ Guidances/UCM201790.pdf

Hankins, M. Still not significant. Psychologically Flawed April 21, 2013. Accessed July 28, 2013 at mchankins.wordpress.com/2013/04/21/ still-not-significant-2/

Ioannidis JPA (2005) Why most published research findings are false. PLoS Med 2:e124

Kairalla JA, Coffey CS, Thomann MA, Muller KE (2012) Adaptive trial designs: a review of barriers and opportunities. Trials 13:145-145

Kerr NL (1998) HARKing: hypothesizing after the results are known. Pers Soc Psychol Rev 2:196-217

Kriegeskorte N, Simmons WK, Bellgowan PSF, Baker CI (2009) Circular analysis in systems neuroscience: the dangers of double dipping. Nat Neurosci 12:535-540

Marino MJ (2014) The use and misuse of statistical methodologies in pharmacology research. Biochem Pharmacol 87:78-92

Motulsky, HJ (2014a) Intuitive Biostatistics 3rd edition, Oxford University Press

Motulsky HJ (2014b) Using simulations to calculate the false discovery rate. Accessed June 1, 2014 at http://www.graphpad.com/support/ faqid/1923/

Motulsky, HJ (2014c). Opinion: Never use the word 'significant' in a scientific paper. Advances in Regenerative Biology 2014, 1: 25155. http://dx.doi.org/10.3402/arb.v1.25155 (in press)

Prinz F, Schlange T, Asadullah K (2011) Believe it or not: how much can we rely on published data on potential drug targets? Nat Rev Drug Discovery 10:712-713. DOI 10.1038/

Simmons J, Nelson L, Simonsohn U (2011) False-positive psychology: undisclosed flexibility in data collection and analysis allow presenting anything as significant. Psychol Sci 22:1359-1366

Ziliak, S, \& McCloskey, DN (2008). The cult of statistical significance: How the standard error costs us jobs, justice, and lives. Ann Arbor: University of Michigan Press. ISBN=0472050079. 